\title{
Organisational and environmental characteristics of residential aged care units providing highly person-centred care: a cross sectional study
}

Karin Sjögren ${ }^{1 *}$ (D) Marie Lindkvist ${ }^{2,3}$, Per-Olof Sandman ${ }^{1,4,6}$, Karin Zingmark ${ }^{4,5}$ and David Edvardsson ${ }^{1,7}$

\begin{abstract}
Background: Few studies have empirically investigated factors that define residential aged care units that are perceived as being highly person-centred. The purpose of this study was to explore factors characterising residential aged care units perceived as being highly person-centred, with a focus on organisational and environmental variables, as well as residents' and staff' characteristics.

Methods: A cross-sectional design was used. Residents $(n=1460)$ and staff $(n=1213)$ data from 151 residential care units were collected, as well as data relating to characteristics of the organisation and environment, and data measuring degree of person-centred care. Participating staff provided self-reported data and conducted proxy ratings on residents. Descriptive and comparative statistics, independent samples t-test, Chi $^{2}$ test, Eta Squared and Phi coefficient were used to analyse data.

Results: Highly person-centred residential aged care units were characterized by having a shared philosophy of care, a satisfactory leadership, interdisciplinary collaboration and social support from colleagues and leaders, a dementiafriendly physical environment, staff having time to spend with residents, and a smaller unit size. Residential aged care units with higher levels of person-centred care had a higher proportion of staff with continuing education in dementia care, and a higher proportion of staff receiving regular supervision, compared to units with lower levels of personcentred care.

Conclusions: It is important to target organisational and environmental factors, such as a shared philosophy of care, staff use of time, the physical environment, interdisciplinary support, and support from leaders and colleagues, to improve person-centred care in residential care units. Managers and leaders seeking to facilitate person-centred care in daily practice need to consider their own role in supporting, encouraging, and supervising staff.
\end{abstract}

Keywords: Care philosophy, Facilitators, Leadership, Person-Centred Care, Physical environment, Residential care facilities, Social support

\footnotetext{
* Correspondence: karin.sjogren@nurs.umu.se; karin.sjogren@umu.se ${ }^{1}$ Department of Nursing, Umeå University, SE-901 87 Umeå, Sweden

Full list of author information is available at the end of the article
} 


\section{Background}

The proportion of old people in the population is increasing in Sweden, and in other western countries. With older age the prevalence of disabilities and chronic diseases, such as dementia increases and consequently also the need for care [1]. This imposes challenges for policy-makers, managers and nursing staff, how to provide care that promotes experiences of well-being, satisfaction, and a meaningful life, in addition to providing merely the symptom control and safe containment of old people and people with dementia.

Research has indicated that residential aged care is at risk of being more task-focused than person-centred [2, 3], that there often is a lack of individually tailored meaningful activities [4-6], and few possibilities for resident choice in daily care and activities $[4,5,7,8]$. It has also been demonstrated that residents and their family members regard independence, autonomy, and community as important, while staff regard these as less valuable [9]. Thus, there is a need for improvement, and person-centred care is increasingly advocated as a model of care that supports holistic well-being for older people and people with dementia in residential aged care [10-12].

There is a growing body of evidence that different forms of PCC-interventions have positive effect on residents' well-being and quality of life (QoL) [13-16], levels of depression [13] and agitation [14, 17, 18], mostly investigated in relation to people with dementia living in residential care facilities. It has also been reported positive effects on staff job satisfaction $[19,20]$, work related stress [20, 21], and burnout [22, 23] from providing person-centred care. However, the evidence to date is not conclusive [24-26], as PCC-studies also report a lack of effect on resident QoL $[17,27]$, as well as a lack of effect on residents agitation [27-29]. Thus, there seems to be a need for further exploration of factors facilitating successful implementation of PCC.

Reported experiences from culture change programmes and interventions in residential aged care facilities, indicate that factors such as effective collaboration and communication, a shared vison of care philosophy, a supportive and enabling management style, informal and formal supervision influence the extent to which person-centred care is really improved in practice [21, 30-35]. A number of authors argue that development of person-centred care in practice should be based on a multilevel approach including intervention actions related to organisation, environment as well as interventions aiming to develop the attitude and knowledge of staff [36-39]. However, a literature review of PCC-interventions [24] indicated that there remain needs to further explore which personal, organisational, and environmental factors that are required to successfully implement PCC.
In theory, person-centred care has been related to a number of factors such as the cognitive and functional abilities of residents [11], as well as to staff's professional and interpersonal competence, commitment and selfknowledge [40]. In addition, theory suggests that having a beneficial psychosocial and physical environment, a supportive and present leadership, and a flexible and supportive organisation can enable person-centred processes [40]. Few studies have empirically investigated which factors that defines residential aged care units that are perceived as being person-centred. Among the few studies that exist, S Caspar, HA Cooke, N O'Rourke and SW MacDonald [41] showed in a cross-sectional study in long term care facilities in Canada, that high quality of inter-professional relationships, support and appreciation from management and necessary time positively predicted a perceived ability to provide person-centred care. A few other studies have reported that person-centred units incorporate psychosocial approaches to behavioural symptoms based on interactional and environmental interpretations, environmental adaptations and reflective individualised social interactions $[42,43]$. It has also been reported that personcentred care is less related to the length of work experience and formal education of staff and leaders [41, 42], but rather to a focus on social care and everyday activities, staff influence and autonomy [44].

To conclude, there is limited research evidence on which factors actually characterise those residential aged care units perceived as offering more person-centred care. Generating valid and reliable knowledge about such factors appear immensely important in guiding practice improvements and development as well as guiding further research and education on this issue. Thus, there is a need to explore personal, organisational and environmental factors that can characterise personcentred residential aged care units, and this study is an attempt to address that gap in knowledge.

\section{Methods \\ Aim}

The aim of this study was to explore factors characterising residential aged care units perceived as being highly person-centred, with a focus on organisational and environmental variables, as well as residents' and staff' variables.

\section{Design}

This study had a cross sectional explorative design. The study is part of a larger project that aimed to explore person-centred care in Swedish residential aged care units, and its association with residents' well-being, staff' perception of their work environment, and organisational factors. Results focusing on residents well-being and staff work environment have been reported elsewhere $[45,46]$. 


\section{Sampling and settings}

Executive officers of municipality-based elderly care, together with dementia care nurses in a nationwide network for dementia care in Sweden, were invited to participate in recruiting residential aged care units for the study. All dementia care nurses and executive officers that were willing to participate received both oral and written information about the study. A contact person was recruited in each unit to oversee the distribution and collection of study surveys.

A total of 87 residential care facilities participated, amounting to 151 individual care units, as facilities could include several autonomous units. The majority of participating care units were special care units for people with dementia (70\%), located in rural $(26 \%)$ and urban $(74 \%)$ regions of Sweden. The unit size ranged from four to 26 residents, and between four and 20 staff members worked on a permanent basis within these units. All residents living in the units were included in the study. Staff were included if they were in permanent employment or on fixed-term contracts.

\section{Data collection}

Data were collected through a resident and a staff survey. The resident survey consisted of demographic variables and established measurement scales on ADL-abilities and cognition. Due to a high expected prevalence of cognitive impairment, proxy ratings were performed. This meant that the staff member who knew each resident best was asked to perform the ratings. The staff survey included demographic variables and established instruments for assessing person-centred care of the unit, perceived social support, as well as some study-specific variables. A total of 1655 resident surveys were distributed, and 1471 were returned (89\%). Eleven resident surveys were excluded due to multiple missing data $(n=1460)$. A total of 1482 staff surveys were distributed, and 1237 were returned (83\%). Twentyfour staff surveys were excluded due to missing data $(n=1213)$. All data was collected in May 2010.

Perceived Person-centred care of the units (PCC) was investigated with the Person-centred Care Assessment Tool (P-CAT) [47, 48]; a scale that measures the extent to which staff perceive care provided as being personcentred. The P-CAT consists of 13 items formulated as statements about the content of care, aspects of the environment and the organisation. A total score is calculated and higher values indicate a higher degree of personcentred care, in a possible range of 13-65. The P-CAT has shown satisfactory estimates of reliability and construct validity in an Australian sample (Cronbach's $\alpha=0.84$ ) [47] and in a Swedish sample (Cronbach's $\alpha=0.75$ ) [48].

To investigate residents' ADL ability five items in the Multi-dimensional Dementia Assessment Scale (MDDAS) [49] was used. Higher scores indicate higher ADL ability in a possible range of 4-24. The prevalence of cognitive capacity was investigated using the Geriatric Rating Scale (GRS) [50], which consists of 27 items concerning the person's ability to orientate. Higher scores indicate a higher ability to orientate in a possible range of $0-27$. Established cut off scores were used to define cognitive impairment or not. A score of less than 24 is considered to indicate cognitive impairment, which correlates with a sensitivity of $90 \%$ and a specificity of $91 \%$ to the cut-off point $24 / 30$ traditionally used in the Mini-mental State Examination (MMSE) [49].

Factors related to the organisation and the environment were investigated by study-specific variables, and validated scales. Five study-specific variables rated on a $100 \mathrm{~mm}$ VAS scale were included; The physical environment is dementia-friendly, We have an interdisciplinary collaboration in care, We have a satisfactory leadership, We have time to spend together with residents, We have a shared philosophy of care. Higher values implied that staff agreed with the statement. Unit size was investigated by reporting the number of residents living in the unit. Whether or not staff received systematic supervision was investigated with a dichotomous item 'yes/no'. The subscale social support of the Swedish DemandControl-Support Questionnaire (DCSQ), [51, 52] was used to investigate the extent to which staff experienced support from co-workers and leaders. The subscale social support consists of 6 items (range of 6-24); a total score was calculated and higher values indicates higher social support. Construct validity and reliability estimates has been reported as satisfactory for the DCSQ (Cronbach's $\alpha=0.83$ ) [52].

Resident characteristics were investigated by studyspecific demographic variables: age; gender; and length of stay in the facility. Staff characteristics were measured by demographic variables: age; gender; years of experience in aged care and work in the current unit, and one dichotomous item regarding continuing education in dementia care.

\section{Analyses}

The IBM SPSS Statistics version $22^{\circ}$ (SPSS. Release 22. Chicago: SPSS Inc.; 2013) was used to analyse the data. The analysis procedure was performed in several steps. First, sample characteristics were explored using descriptive and comparative statistics. Secondly, PCC was aggregated on unit level (mean value for the unit), and divided into two groups; higher PCC including care units in the highest median $(n=74$, PCC scores $\geq 48.79)$ and lower PCC including care units in the lower median ( $n=77$, PCC scores $\leq 48.80)$. Thirdly, differences in resident characteristics, staff characteristics, environmental and organizational factors between units with higher and lower levels of PCC were explored using independent samples t-test for continuous variables and $\mathrm{Chi}^{2}$ test 
for ordinal and nominal variables. Eta Squared and Phi coefficient were used to calculate effect size.

\section{Results}

\section{Description of the sample}

The mean age of residents $(n=1460)$ was 85 years $(\mathrm{SD} \pm 8)$, and their mean stay in the care unit was about 2.6 years $(\mathrm{SD} \pm 3)$. The majority were women $(70 \%)$ and $88 \%$ had cognitive impairment. Ten per cent of residents could dress independently, $5 \%$ could manage their personal hygiene independently, and $49 \%$ could eat and drink independently. The mean age of staff $(n=1213)$ was 46 years $(\mathrm{SD} \pm 11)$, they had worked in aged care for about 16 years $(S D \pm 10)$ and in the same unit for about 7.6 years $(\mathrm{SD} \pm 7)$. The majority were women (95\%), qualified as enrolled nurses (80\%), and a slight majority $(56 \%)$ had some continuing education in dementia care. The sample characteristics of residents and staff in included care units are shown in Tables 1 and 2.

There were no significant differences related to residents' characteristics (gender, aged, cognitive impairment, ADLcapacity, length of stay in facility) between units with high PCC (PCC $\geq 48.79)$ and units with low PCC ( $\leq 48.80)$ (see Table 3). For staff characteristics, it was shown that in units with high PCC a higher proportion of staff (61.2\%) had some additional education in dementia care compared to units with low PCC $(51.1 \%, p=0.001$, $p h i-0.1)$. No significant differences were found related to other staff characteristics (gender, aged, experience from aged care).

When comparing organisational and environmental variables, it was found that, in units with higher levels of PCC, the number of residents per unit were lower $(13.7 \pm 9.7)$ compared to in units with lower PCC $(14.8 \pm 9.3, p=0.05$, $\eta=0.003)$. The variables interdisciplinary collaboration $(p<0.001, \eta=0.041)$, satisfactory leadership $(p<0.001$, $\eta=0.107)$, a dementia-friendly physical environment, $(p<0.001, \eta=0.045)$, time to spend with residents

Table 1 Characteristics of the sample (residents)

\begin{tabular}{llr}
\hline & $\mathrm{n}^{\mathrm{a}}(\%)$ & $\mathrm{m}(\mathrm{SD})$ \\
\hline Sex & & \\
Women & $375(30.1)$ & \\
Age, years & $870(69.9)$ & \\
Time in facility, years & & \\
$\quad$ Cognitive impairment & & \\
Yes & & \\
No & $1261(2.5)$ \\
Normal ability to dress on own initiative & $123(98.3)$ & \\
Manages personal hygiene on own initiative & $63(5.0)$ \\
Eats and drinks independently & $611(48.9)$ \\
\hline
\end{tabular}

${ }^{a} \mathrm{n}$ does not always add up to 1460 in all variables due to missing items
Table 2 Characteristics of the sample (staff)

\begin{tabular}{lll}
\hline & $\mathrm{n}^{\mathrm{a}}(\%)$ & $\mathrm{m}( \pm \mathrm{SD})$ \\
\hline Sex & & \\
Men & $56(4.8)$ & \\
Age (Years) & $1105(95.2$ & \\
Years of experience in aged care & & $15.9(11.2)$ \\
Years of work in this facility & & $7.6(6.9)$ \\
Qualifications & & \\
$\quad$ Registered nurses & & \\
$\quad$ Enrolled nurses & $35(3.1)$ & \\
$\quad$ Nurses' assistants & $910(80.1)$ & \\
$\quad$ No formal qualifications & $120(10.6)$ & \\
$\quad$ Other education & $42(3.7)$ & \\
Regular supervision & $29(2.6)$ \\
Additional education in dementia care & $598(56.0)$ &
\end{tabular}

${ }^{a} \mathrm{n}$ does not always add up to 1213 in all variables due to missing items

$(p<0.001, \eta=0.108)$, a shared philosophy of care $(p<0.001, \eta=0.090)$, and social support $(p<0.001$, $\eta=0.069$ ), were rated significantly higher in units with higher PCC compared to units with lower PCC. In addition, the number of staff who received regular supervision was higher in units with high levels of PCC compared to in units with low levels of PCC $(p=0.005, p h i-0.09)$ (Table 4).

In this sample, the Cronbach alpha estimates were 0.75 for the P-CAT, 0.84 for the Social support subscale, and 0.95 for the GRS.

\section{Discussion}

The purpose of this study was to explore which factors characterise residential aged care units perceived as being highly person-centred. The results indicate that highly person-centred residential aged care units were characterized by a number of organisational and environmental facilitating factors, i.e. a shared philosophy of care, time to spend with residents, social support from colleagues and leaders, a dementia-friendly physical environment, interdisciplinary collaboration, regular supervision of staff, and a smaller unit size, one staff characteristic; continuing education in dementia care and no particular resident characteristics.

A shared philosophy of care characterises highly personcentred residential aged care units. This finding confirms the theoretical proposal [37] that a strong shared value base is an important prerequisite for person-centred care, implying that leaders and managers should work with their teams to develop a shared vision of person-centred care [40]. This finding is also supported by experiences from PCC intervention studies and practise development programmes, in which managers and staff describe that the values and ideas of person-centred care need to be embedded in the whole organisation in order to make person-centred care happen 
Table 3 Comparison of residents and staff characteristics between units with high and low levels of PCC

\begin{tabular}{|c|c|c|c|c|c|c|}
\hline & \multicolumn{2}{|l|}{ Low PCC } & \multirow{2}{*}{$\begin{array}{l}\text { High PCC } \\
M(s d)\end{array}$} & \multirow[b]{2}{*}{$\mathrm{n}(\%)$} & \multirow[b]{2}{*}{$p$-value } & \multirow[b]{2}{*}{ Effect size } \\
\hline & $\overline{M(s d)}$ & $n(\%)$ & & & & \\
\hline \multicolumn{7}{|l|}{ Residents } \\
\hline \multicolumn{7}{|l|}{ Gender } \\
\hline Women & & $518(69.2)$ & & $467(67.3)$ & & \\
\hline Men & & $231(30.8)$ & & $227(32.7)$ & 0.45 & \\
\hline Age & $84.5(3.3)$ & & $84.9(2.8)$ & & 0.40 & \\
\hline Cognitive impairment & & & $644(87.6)$ & $617(89.0)$ & 0.41 & \\
\hline ADL-ability & $13.9(6.1)$ & & $14.3(6.0)$ & & 0.20 & \\
\hline Time for stay in this facility (months) & $32.1(29.7)$ & & $31.0(31.4)$ & & 0.50 & \\
\hline \multicolumn{7}{|l|}{ Staff } \\
\hline \multicolumn{7}{|l|}{ Gender } \\
\hline Women & & $580(94.2)$ & & $564(96.1)$ & & \\
\hline Men & & $36(5.8)$ & & $23(3.9)$ & 0.10 & \\
\hline Age & $45.9(11.5)$ & & $45.7(10.9)$ & & 0.75 & \\
\hline Years of experience in aged care & $15.5(10.4)$ & & $16.0(10.3)$ & & 0.39 & \\
\hline Years of work in this facility & $7.3(6.5)$ & & $7.9(7.4)$ & & 0.10 & \\
\hline Additional education in dementia care & & $291(51.1)$ & & $328(61.2)$ & 0.001 & $-0.1^{\mathrm{a}}$ \\
\hline
\end{tabular}

$[31-33,53]$. To our knowledge, the influence of having a shared philosophy of care as rated by direct care staff, has not been operationalized and studied before in relation to PCC. In a study from the U.S, J Banaszak-Holl, NG Castle, M Lin and G Spreitzer [54] found that nursing homes involved in person-centred development programmes did not report higher person-centred cultural values than other nursing homes. However, as no objective measurement of person-centered care was conducted in the nursing homes included in their study, the impact of cultural values on person-centred care remain unknown. Further studies that explore the content and process in creating shared personcentred care values in nursing homes would be valuable. Questions such as how to facilitate shared values of personcentred care and how such core values are translated into actual day-to-day interactions, priorities and activities need further study.

The results indicate that perceptions of having time as a staff member to just be together with residents was a factor that characterized person-centred residential aged care units. This aspect could be a part of the shared values of PCC, but it can also be a matter of perceived workload if staff perceive themselves as having sufficient time or freedom to prioritise spending time just being with residents. Although it has been reported that staff perceive that a person-centred approach in care takes more time and resources [41], it has also been reported that person-centred care is associated with a better use of time $[55,56]$, creating more possibilities for positive interactions and encounters.

Table 4 Comparison of organisational and environmental factors between units with high and low levels of PCC

\begin{tabular}{|c|c|c|c|c|c|c|}
\hline & \multicolumn{2}{|l|}{ Low PCC } & \multicolumn{2}{|l|}{ High PCC } & \multirow[b]{2}{*}{$p$-value } & \multirow[b]{2}{*}{ Effect size } \\
\hline & $\overline{M(s d)}$ & $N(\%)$ & $\bar{M}(\mathrm{sd})$ & $N(\%)$ & & \\
\hline Unit size (number of residents) & $14.8(9.3)$ & & $13.7(9.7)$ & & 0.05 & $0.003^{a}$ \\
\hline Interdisciplinary collaboration & $69.0(28.5)$ & & $79.7(23.6)$ & & $<0.001$ & $0.041^{\mathrm{a}}$ \\
\hline Leadership & $60.2(27.3)$ & & $77.1(21.6)$ & & $<0.001$ & $0.107^{\mathrm{a}}$ \\
\hline Dementia friendly physical environment & $50.2(29.8)$ & & $63.1(29.9)$ & & $<0.001$ & $0.045^{\mathrm{a}}$ \\
\hline Time to spend being with residents & $53.6(27.5)$ & & $71.8(24.7)$ & & $<0.001$ & $0.108^{\mathrm{a}}$ \\
\hline Shared philosophy of care & $70.0(24.8)$ & & $83.7(18.3)$ & & $<0.001$ & $0.090^{a}$ \\
\hline Social support & $19.2(3.0)$ & & $20.7(2.6)$ & & $<0.001$ & $0.069^{\mathrm{a}}$ \\
\hline Regular supervision & & $102(17.4)$ & & $139(24.3)$ & 0.005 & $-0.09^{b}$ \\
\hline
\end{tabular}

${ }^{\mathrm{a}}$ Eta squared, ${ }^{\mathrm{b}}$ Phi coefficient 
Few studies have systematically investigated time use in PCC-interventions [16, 17]. L Chenoweth, MT King, YH Jeon, H Brodaty, J Stein-Parbury, R Norman, M Haas and G Luscombe [17] reported the cost for implementation of two PCC-models (Dementia Care Mapping and PCCeducation), which included time for education and training and the time staff used on PCC activities that were related to the intervention. It was demonstrated that education in PCC was more cost effective than using Dementia Care Mapping in relation to reduction in agitation scores, and there was a decreased cost per unit reduction in agitation scores over time for PCC-education. As agitation is distressing and drives cost for increased staff time, the results of the study [17] may be interpreted to indicate that PCC may take additional time initially, with subsequent time and cost savings in the long term.

Another aspect, reported in intervention studies, is that care staff describe that competing demands in addition to lack of time is a barrier to PCC [30]. This highlights the importance for managers and leaders to support direct care workers in making priorities in daily care that facilitates person-centred care. Again this seems related to an overall philosophy of care and if such an explicit or implicit philosophy contains a focus on completing tasks or facilitating well-being.

Social support from colleagues and leaders and satisfactory leadership were also factors related to person-centred care. Theoretical models have previously described the importance of a supportive leader who takes responsibility for an effective, sustainable person-centred culture change and quality improvement in residential aged care facilities $[40,57,58]$. However, few research studies have investigated the importance of leadership and leadership support in enhancing person-centred care. PV Hunter, T Hadjistavropoulos, L Thorpe, LM Lix and DC Malloy [59] showed in an explorative study in two Canadian longterm care homes that structural supervisory and organizational support was not related to different dimensions of PCC, while leaders' support for collaboration in care was related to different aspects of PCC. In a recently published controlled trial, Y-H Jeon, JM Simpson, Z Li, MM Cunich, TH Thomas, L Chenoweth and HL Kendig [60] reported no change in person-centred care after a 12month leadership development programme. A Backman, K Sjögren, M Lindkvist, $\mathrm{H}$ Lövheim and D Edvardsson [61] reported of positive relationships between effective leadership behaviours and person-centred care. This study contributes to the existing research evidence, by suggesting that the support from managers and leaders is important for facilitating person-centred care.

The results of this study also indicate that a physical environment experienced as dementia-friendly is a factor related to person-centred care. Others have conceptualised a person-centred physical environment as facilitating the independence of residents, positive interactions between residents and staff, and resident participation in collaborative work [62], with the potential to support or hinder residents' autonomy [59], and allowing residents to be an active participant in everyday life rather than a passive recipient of care [63]. This is supported by results from intervention studies showing that person-centred changes to the environment have had positive effect on QoL and agitation for residents with dementia [16, 64].

The results of this study indicate that the physical environment is an important and meaningful factor in person-centred practice which supports theoretical models proposing that, in order to support personcentred care in residential aged care facilities, it is necessary to change environments to match the competence of the individual [39].

\section{Methodological considerations}

The cross-sectional nature of this study limits causal interpretations of the results. Further studies with interventional and longitudinal designs are needed to further investigate causality. Another limitation is that the study relies on self-reported (staff) data of person-centred care. It is not known whether or not ratings correspond to what actually happens in practice, and thus further studies that combine staff ratings, and family members ratings with observations would be valuable so as to investigate the extent to which behaviours correspond to what is reported. The choice of measurement scales to use when evaluating person-centred care of the unit may need consideration. The P-CAT as used in this study is a short and comprehensive scale including concrete items that are formulated to capture essential elements of person-centred care in units, and it was found appropriate to use as a numerical way to differentiate and compare units with higher and lower degrees of personcentred care. However, it would be valuable to further investigate factors that contribute to define highly person-centred units, including multiple measures of person-centred care to gain further knowledge that can guide PCC-development work. The P-CAT is limited to staff perceptions of person-centred care, and thus other perspectives on this would be valuable.

Nevertheless, the study draws on a large sample from a geographically varied area of Sweden, includes both resident and staff ratings, and makes contemporary relevant analyses of empirical relationships between nursing variables based on extensive nursing theory. Thus, the study makes a small but significant contribution to the literature on the sometimes elusive concept of personcentred care in nursing, by providing empirical data that further contributes to our understanding of what characterises residential aged care units perceived as being person-centred. 
A strength of this study is the large sample size of participating units. A network of dementia care nurses in Sweden assisted in recruiting units for this study. In Sweden, dementia care nurses are responsible for dementia care in the municipalities, commonly well-known by staff and managers in residential aged care, and their engagement was instrumental in encouraging facilities and units to participate in the study. Furthermore, unit scores on variables such as person-centred care and social support, were promised and later reported back to the unit together with a short text on how to interpret and understand the scores and results. This reporting back of scores and results is likely to have served as an encouragement to participate in the study, as it provided managers and staff measurable and comparable data as a baseline for their own practice development work, as well as an entry point and incitement to continue with repeated measures and practice development work.

\section{Conclusions}

The knowledge concerning facilitating factors related to person-centred care is mainly based on theoretical discussions and models. This study indicates that highly person-centred residential aged care units can be characterised by a number of organisational and environmental factors, which can be interpreted as facilitators for person-centred care. The study contributes to the emerging research knowledge by suggesting areas that can be assessed and targeted in efforts to facilitate personcentred care in residential aged care units.

It seems important to create a shared philosophy of care among all those involved - managers, care workers, residents and family members - and to enhance care workers in making priorities that increase time and possibilities for social interactions and encounters. Furthermore, changing the physical environment so that it matches residents' abilities and preferences, and prioritizing support and collaboration between staff members is also important to increase person-centred care in residential care units. Managers and leaders seeking to improve person-centred care will need to consider their own role in supporting, encouraging, and supervising staff in this practice improvement.

\section{Abbreviations}

ADL: Activities of Daily Living; PCC: Person-Centred Care; QoL: Quality of Life

\section{Acknowledgements}

We acknowledge the contribution of Nurses in the network for dementia care in Sweden for assisting in data collection.

\section{Funding}

This study was funded by Swedish Brainpower and Umeå University.

\section{Availability of data and materials}

The datasets analysed during the current study available from the corresponding author on reasonable request.

\section{Authors' contributions}

KS, designed the study, collected, analysed and interpreted the data, and was a major contributor in writing the manuscript. ML contributed by analysing and interpreting the data, and in writing the manuscript. P-O S designed the study and contributed in writing the manuscript. KZ contributed in writing the manuscript, DE was the project leader, designed the study, participated in analysing and interpreting the data, and in writing the manuscript. All authors read and approved the final manuscript.

\section{Ethics approval and consent to participate}

The study was approved by the Regional Ethical Review Board in Umeå, Sweden (Dnr 08-195 M, Dnr 2010-135-32).

\section{Consent for publication}

Not applicable.

\section{Competing interests}

The authors declare that they have no competing interests.

\section{Publisher's Note}

Springer Nature remains neutral with regard to jurisdictional claims in published maps and institutional affiliations.

\section{Author details}

'Department of Nursing, Umeå University, SE-901 87 Umeå, Sweden. 2Department of Statistics, Umeå School of Business and Economics, Umeå University, Umeå, Sweden. ${ }^{3}$ Epidemiology and Global Health, Department of Public Health and Clinical Medicine, Umeå university, Umeå, Sweden. ${ }^{4}$ Division of Nursing, Department of Health Science, Luleå University of Technology, Luleå, Sweden. ${ }^{5}$ Region of Norrbotten, Luleå, Sweden. ${ }^{6}$ Division of Caring Sciences, Department of Neurobiology, Care Sciences and Society (NVS), Karolinska Institutet, Stockholm, Sweden. ${ }^{7}$ School of Nursing and Midwifery, La Trobe University, Melbourne, Australia.

Received: 17 January 2017 Accepted: 1 August 2017

Published online: 10 August 2017

\section{References}

1. Wimo A, Jönsson L, Bond J, Prince M, Winblad B. The worldwide economic impact of dementia 2010. Alzheimers Dement. 2013;9(1):1-11. e13

2. Furåker $\mathrm{C}$, Nilsson A. The competence of certified nurse assistants caring for persons with dementia diseases in residential facilities. J Psychiatr Ment Health Nurs. 2009;16(2):146-52.

3. Beck I, Törnquist A, Broström L, Edberg A-K. Having to focus on doing rather than being-Nurse assistants' experience of palliative care in municipal residential care settings. Int J Nurs Stud. 2012;49(4):455-64.

4. Kirkevold $\varnothing$, Engedal $K$. The quality of care in Norwegian nursing homes. Scand J Caring Sci. 2006;20(2):177-83.

5. Berglund AL. Satisfaction with caring and living conditions in nursing homes: Views of elderly persons, next of kin and staff members. Int J Nurs Pract. 2007;13(1):46-51

6. Wood W, Womack J, Hooper B. Dying of boredom: An exploratory case study of time use, apparent affect, and routine activity situations on two Alzheimer's special care units. The American Journal of Occupational Therapy. 2009;63(3):337-50.

7. Schnelle JF, Bertrand R, Hurd D, White A, Squires D, Feuerberg M, Hickey K, Simmons SF. Resident choice and the survey process: The need for standardized observation and transparency. The Gerontologist. 2009;49(4):517-24.

8. Luff $R$, Ellmers T, Eyers I, Young E, Arber S. Time spent in bed at night by carehome residents: choice or compromise? Ageing Soc. 2011;31(7):1229-50.

9. White HK, Corazzini K, Twersky J, Buhr G, McConnell E, Weiner MF, ColónEmeric CS. Prioritizing culture change in nursing homes: perspectives of residents, staff, and family members. J Am Geriatr Soc. 2012;60(3):525-31.

10. Epp TD. Person-centred dementia care: A vision to be refined. The Canadian Alzheimer Disease Review. 2003;5(3):14-9.

11. Dewing J. Concerns relating to the application of frameworks to promote person-centredness in nursing with older people. J Clin Nurs. 2004:13(3a):39-44

12. Edvardsson D, Winblad B, Sandman PO. Person-centred care of people with severe Alzheimer's disease: current status and ways forward. Lancet Neurol. 2008;7(4):362-7. 
13. Brooker DJ, Woolley RJ, Lee D. Enriching opportunities for people living with dementia in nursing homes: An evaluation of a multi-level activitybased model of care. Aging and Mental Health. 2007;11(4):361-70.

14. Cohen-Mansfield J, Thein K, Marx MS, Dakheel-Ali M, Freedman L. Efficacy of nonpharmacologic interventions for agitation in advanced dementia: a randomized, placebo-controlled trial. The Journal of Clinical Psychiatry. 2012; 73(9):1255-61.

15. Rokstad AMM, Røsvik J, Kirkevold Ø, Selbaek G, Saltyte Benth J, Engedal K. The Effect of Person-Centred Dementia Care to Prevent Agitation and Other Neuropsychiatric Symptoms and Enhance Quality of Life in Nursing Home Patients: A 10-Month Randomized Controlled Trial. Dement Geriatr Cogn Disord. 2013;36(5-6):340-53.

16. Chenoweth L, Forbes I, Fleming R, King MT, Stein-Parbury J, Luscombe G, Kenny $P$, Jeon $Y-H$, Haas M, Brodaty $H$. PerCEN: a cluster randomized controlled trial of person-centered residential care and environment for people with dementia. Int Psychogeriatr. 2014;26(07):1147-60.

17. Chenoweth L, King MT, Jeon YH, Brodaty H, Stein-Parbury J, Norman R, Haas M, Luscombe G. Caring for Aged Dementia Care Resident Study (CADRES) of person-centred care, dementia-care mapping, and usual care in dementia: a cluster-randomised trial. Lancet Neurol. 2009;8(4):317-25.

18. Fossey J, Ballard C, Juszczak E, James I, Alder N, Jacoby R, Howard R. Effect of enhanced psychosocial care on antipsychotic use in nursing home residents with severe dementia: cluster randomised trial. BMJ. 2006;332(7544):756-61.

19. McCormack B, Dewing J, Breslin L, Coyne-Nevin A, Kennedy K, Manning M, Peelo-Kilroe L, Tobin C, Slater P. Developing person-centred practice: nursing outcomes arising from changes to the care environment in residential settings for older people. Int J Older People Nursing. 2010;5(2):93-107.

20. van Weert JC, van Dulmen AM, Spreeuwenberg PM, Bensing JM, Ribbe MW. The effects of the implementation of snoezelen on the quality of working life in psychogeriatric care. Int Psychogeriatr. 2005;17(03):407-27.

21. Edvardsson D, Sandman P, Borell L. Implementing national guidelines for person-centered care of people with dementia in residential aged care: effects on perceived person-centeredness, staff strain, and stress of conscience. Int Psychogeriatr. 2014;26(07):1171-9.

22. Passalacqua SA, Harwood J. VIPS Communication Skills Training for Paraprofessional Dementia Caregivers: An Intervention to Increase PersonCentered Dementia Care. Clin Gerontol. 2012;35(5):425-45.

23. Jeon Y-H, Luscombe G, Chenoweth L, Stein-Parbury J, Brodaty H, King M, Haas M. Staff outcomes from the caring for aged dementia care resident study (CADRES): a cluster randomised trial. Int J Nurs Stud. 2012;49(5):508-18.

24. Brownie $S$, Nancarrow S. Effects of person-centered care on residents and staff in aged-care facilities: a systematic review. Clin Interv Aging. 2013;8:1-10.

25. Barbosa A, Sousa L, Nolan M, Figueiredo D. Effects of person-centered care approaches to dementia care on staff: a systematic review. American Journal of Alzheimer's Disease \& Other Dementias ${ }^{\oplus}$. 2015;30(8):713-22.

26. Pol-Grevelink A, Jukema J, Smits C. Person-centred care and job satisfaction of caregivers in nursing homes: a systematic review of the impact of different forms of person-centred care on various dimensions of job satisfaction. International Journal of Geriatric Psychiatry. 2012;27:219-29.

27. Dichter MN, Quasdorf T, Schwab CGG, Trutschel D, Haastert B, Riesner C, Bartholomeyczik S, Halek M. Dementia care mapping: effects on residents' quality of life and challenging behavior in German nursing homes. A quasiexperimental trial. Int Psychogeriatr. 2015;27(11):1875-92.

28. van de Ven G, Draskovic I, Adang EM, Donders R, Zuidema SU, Koopmans RT, Vernooij-Dassen MJ. Effects of dementia-care mapping on residents and staff of care homes: a pragmatic cluster-randomised controlled trial. PLoS One. 2013;8(7):e67325. doi:10.1371/journal.pone.0067325.

29. Sidani S, Streiner D, LeClerc C. Evaluating the effectiveness of the abilitiesfocused approach to morning care of people with dementia. Int J Older People Nurs. 2012;7(1):37-45.

30. Brooker DJ, Woolley RJ. Enriching opportunities for people living with dementia: The development of a blueprint for a sustainable activity-based model. Aging and Mental Health. 2007;11(4):371-83.

31. Leutz W, Bishop CE, Dodson L. Role for a Labor-Management Partnership in Nursing Home Person-Centered Care. The Gerontologist. 2010;50(3):340-51.

32. Kirkley C, Bamford C, Poole M, Arksey H, Hughes J, Bond J. The impact of organisational culture on the delivery of person-centred care in services providing respite care and short breaks for people with dementia. Health \& Social Care in the Community. 2011;19(4):438-48.
33. Røsvik J, Kirkevold M, Engedal K, Brooker D, Kirkevold Ø. A model for using the VIPS framework for person-centred care for persons with dementia in nursing homes: a qualitative evaluative study. Int J Older People Nursing. 2011;6(3):227-36.

34. Rosemond CA, Hanson LC, Ennett ST, Schenck AP, Weiner BJ. Implementing person-centered care in nursing homes. Health Care Manag Rev. 2012;37(3):257-66.

35. Vikström S, Sandman P-O, Stenwall E, Boström A-M, Saarnio L, Kindblom K, Edvardsson D, Borell L. A model for implementing guidelines for personcentered care in a nursing home setting. Int Psychogeriatr. 2015;27(01):49-59.

36. Kitwood TM. Dementia reconsidered : the person comes first. Buckingham: Open University Press; 1997.

37. Brooker D. What is person-centred care in dementia? Rev Clin Gerontol. 2004;13(3):215-22.

38. Robinson GE, Gallagher A. Culture change impacts quality of life for nursing home residents. Top Clin Nutr. 2008;23(2):120-30.

39. McGilton KS, Heath H, Chu CH, Boström AM, Mueller C, Boscart VM, McKenzie-Green B, Moghabghab R, Bowers B. Moving the agenda forward: a person-centred framework in long-term care. Int J Older People Nursing. 2012;7(4):303-9.

40. McCormack B, McCance T. Person-centred nursing : theory and practice. Chichester, West Sussex: Wiley-Blackwell; 2010.

41. Caspar S, Cooke HA, O'Rourke N, MacDonald SW. Influence of Individual and Contextual Characteristics on the Provision of Individualized Care in LongTerm Care Facilities. The Gerontologist. 2013;53(5):790-800.

42. Zimmerman S, Sloane PD, Williams CS, Reed PS, Preisser JS, Eckert JK, Boustani M, Dobbs D. Dementia care and quality of life in assisted living and nursing homes. The Gerontologist. 2005;45(suppl 1):133-46.

43. Terada S, Oshima E, Yokota O, Ikeda C, Nagao S, Takeda N, Sasaki K, Uchitomi Y. Person-centered care and quality of life of patients with dementia in long-term care facilities. Psychiatry Res. 2013;205(1-2):103-8.

44. Bonifas RP. Nursing Home Work Environment Characteristics: Associated Outcomes in Psythosocial Care. Health Care Financing Review. 2008;30(2):19-33.

45. Sjögren K, Lindkvist M, Sandman PO, Zingmark K, Edvardsson D. Personcentredness and its association with resident well-being in dementia care units. J Adv Nurs. 2013;69(10):2196-206.

46. Sjögren $K$, Lindkvist M, Sandman PO, Zingmark K, Edvardsson D. To what extent is the work environment of staff related to person-centred care? A cross-sectional study of residential aged care. J Clin Nurs. 2015;24(9-10):1310-9.

47. Edvardsson D, Fetherstonhaugh D, Nay R, Gibson S. Development and initial testing of the Person-centered Care Assessment Tool (P-CAT). Int Psychogeriatr. 2010;22(1):101-8.

48. Sjögren K, Lindkvist M, Sandman PO, Zingmark K, Edvardsson D. Psychometric evaluation of the Swedish version of the Person-Centered Care Assessment Tool (P-CAT). Int Psychogeriatr. 2012;24(3):406-15.

49. Sandman $P$, Adolfsson R, Norberg A, Nyström L, Winblad B. Long-term care of the elderly. A descriptive study of 3600 institutionalized patients in the county of Vasterbotten, Sweden. Comprehensive gerontology Section A, Clinical and laboratory sciences. 1988;2(3):120-33.

50. Gottfries C, Gottfries I, Roos B. The investigation of homovanillic acid in the human brain and its correlation to senile dementia. Br J Psychiatry. 1969; 115(522):563-74.

51. Theorell T, Karasek RA. Current issues relating to psychosocial job strain and cardiovascular disease research. J Occup Health Psychol. 1996;1(1):9-26.

52. Sanne B, Torp S, Mykletun A, Dahl AA. The Swedish Demand-Control-Support Questionnaire (DCSQ): Factor structure, item analyses, and internal consistency in a large population. Scandinavian journal of public health. 2005;33(3):166-74.

53. Williams J, Hadjistavropoulos T, Ghandehari O, Yao X, Lix L. An evaluation of a person-centred care programme for long-term care facilities. Ageing Soc. 2015;35(03):457-88.

54. Banaszak-Holl J, Castle NG, Lin M, Spreitzer G. An assessment of cultural values and resident-centered culture change in US nursing facilities. Health Care Manag Rev. 2013;38(4):295-305.

55. Kontos PC, Mitchell GJ, Mistry B, Ballon B. Using drama to improve personcentred dementia care. Int J Older People Nursing. 2010;5(2):159-68.

56. Palese A, Granzotto D, Broll MG, Carlesso N. From health organizationcentred standardization work process to a personhood-centred care process in an Italian nursing home: effectiveness on bowel elimination model. Int J Older People Nursing. 2010;5(2):179-87.

57. Jeon YH, Conway J, Chenoweth L, Weise J, Thomas TH, Williams A. Validation of a clinical leadership qualities framework for managers in aged care: a Delphi study. J Clin Nurs. 2015;24(7-8):999-1010. 
58. Lynch BM, McCormack B, McCance T. Development of a model of situational leadership in residential care for older people. J Nurs Manag. 2011;19(8):1058-69.

59. Hunter PV, Hadjistavropoulos T, Thorpe L, Lix LM, Malloy DC. The influence of individual and organizational factors on person-centred dementia care. Aging Ment Health. 2015;20(7):700-8.

60. Jeon Y-H, Simpson JM, Li Z, Cunich MM, Thomas TH, Chenoweth L, Kendig HL. Cluster Randomized Controlled Trial of An Aged Care Specific Leadership and Management Program to Improve Work Environment, Staff Turnover, and Care Quality. J Am Med Dir Assoc. 2015;16(7):629. e19-629.e28

61. Backman A, Sjögren K, Lindkvist M, Lövheim H, Edvardsson D. Towards person-centredness in aged care-exploring the impact of leadership. J Nurs Manag. 2016;24:766-74.

62. Morgan-Brown M, Newton R, Ormerod M. Engaging life in two Irish nursing home units for people with dementia: Quantitative comparisons before and after implementing household environments. Aging and Mental Health. 2012:17(1):57-65.

63. Davis S, Byers S, Nay R, Koch S. Guiding design of dementia friendly environments in residential care settings: Considering the living experiences. Dementia. 2009;8(2):185-203.

64. Kane RA, Lum TY, Cutler LJ, Degenholtz HB, Yu TC. Resident Outcomes in Small-House Nursing Homes: A Longitudinal Evaluation of the Initial Green House Program. J Am Geriatr Soc. 2007;55(6):832-9.

\section{Submit your next manuscript to BioMed Central and we will help you at every step:}

- We accept pre-submission inquiries

- Our selector tool helps you to find the most relevant journal

- We provide round the clock customer support

- Convenient online submission

- Thorough peer review

- Inclusion in PubMed and all major indexing services

- Maximum visibility for your research

Submit your manuscript at www.biomedcentral.com/submit 